\section{Telekom kündigt Praxen die Router-Verträge}

\author{
Die Deutsche Telekom hat den Router "Medical Exchange" eingestellt. \\ Betroffene Praxen müssen nun einen neuen Provider suchen.
}

$\ddot{A}$ rzte, die über den Router der Deutschen Telekom Zugang zum sicheren Netz der KVen bekommen haben, müssen sich einen neuen Provider suchen. Das Unternehmen hat das Gerät „Medical Exchange“ zum 21. Oktober eingestellt und die Verträge mit rund 2.200 Ärzten gekündigt. Die Zertifizierung für den Router ist zum 30. Juni abgelaufen.

Der Vorstand der KV Westfalen-Lippe Dr. Thomas Kriedel hat das Unternehmen für sein Verhalten scharf kritisiert. „Es ist ein Unding, Kunden so hängen zu lassen“, sagte Kriedel auf der Vertreterversammlung der KV Westfalen-Lippe in Dortmund. In Westfalen-Lippe sind nach seinen Angaben 750 Praxen mit 1.000 Ärzten von der Entscheidung betroffen. Sie müssen sich jetzt um einen neuen Provider kümmern.

\section{Sicherheitsanforderungen zu hoch}

Wie die anderen Anbieter auch, hätte die Telekom den Router für das sichere Netz der KVen - auch bekannt als KV-Safenet - gemäß den Anforderungen des Bundesamts für Sicherheit in der Informationstechnik rezertifizieren lassen müssen. Das Amt hatte ein Sicherheits-Update gefordert. Darauf hat das Unternehmen jedoch verzichtet und den Kunden deshalb keine neue Hardware geliefert. „Die Ärzte haben sich auf die Telekom verlassen“, monierte Kriedel. Dieses Geschäftsgebaren sollten die Ärzte im Auge haben, wenn sie demnächst mal wieder einen Anbieter im Bereich der Telekommunikation brauchen, findet er. „Wir können den Unmut der Ärzte verstehen und bedauern sehr, dass wir uns zu diesem Schritt entschließen mussten, sehen aber nach Abwägung aller Sachverhalte keine andere Lösung", erklärte Dirk Becker, Pressesprecher der Deutschen Telekom. In der vorgegebenen Zeit habe Medical Exchange nicht an die erhöhten Sicherheitsanforderungen angepasst werden können.

\section{Eventuell Ersatz für Wechselkosten}

Die Einstellung zum 21. Oktober bedeutete, dass die Ärzte ihre Abrechnung für das dritte Quartal noch mit dem Router über das sichere Netz der KVen haben abwickeln können. Die Telekom empfahl jedoch laut Becker, gleichzeitig ein alternatives Angebot eines zertifizierten sowie bewährten KV-Safenet-Anbieters wahrzunehmen, sodass die Kontinuität sichergestellt sei.

Die Kassenärztliche Bundesvereinigung (KBV) weist betroffene Ärzte darauf hin, dass sie für den Wechsel zu einem neuen KV-Safenet-Provider laut der Richtlinie der KBV gegebenenfalls Wechselkosten bei der Telekom geltend machen können. „Ausschlaggebend dafür sind jedoch die individuellen Verträge zwischen Praxis und Telekom als Anbieter", heißt es in einer Stellungnahme der KBV. Sie rät den betroffenen Praxen, ihre Verträge darauf hin zu überprüfen und hat online Informationen zu anderen Providern zusammengestellt.

Ilse Schlingensiepen

\section{KBV-Informationen}

zu alternativen Providern:

http://www.kbv.de/html/7146.php
Patienten, die mindestens drei verordnete Medikamente gleichzeitig anwenden, haben nun Anspruch auf den bundeseinheitlichen Medikationsplan (BMP). Der Softwareanbieter Indamed will Ärzten den Plan kostenfrei im Rahmen des normalen Quartal-Updates zur Verfügung stellen. Der bereits in der Software "Medical Office" vorhandene Medikationsplan könne parallel genutzt werden. Schon erstellte Praxismedikationspläne blieben unverändert, die Daten für den BMP sollen per Mausklick übernommen werden können. Außerdem will Indamed die Handhabe des QR-Codes möglichst flexibel gestalten: Anwender könnten den QR-Code künftig nach Wunsch mit einem Handbarcodescanner, einem Dokumentenscanner, einem Smartphone oder einer Webcam in ihr System einlesen und über den aktuellen Medikationsplan nicht nur in der Praxis, sondern auch unterwegs beim Hausbesuch verfügen.

RebekkaHöhl

\section{Microsoft startet Cloud in Deutschland}

Microsoft hat am 21. September für Geschäftskunden in Europa ein Paket von Cloud-Diensten gestartet, mit dem rechtliche Vorbehalte gegen die Nutzung aus dem Weg geräumt werden sollen. Unter dem Namen „Azure Deutschland" werden Rechenleistungen, Datenspeicherung im Internet und komplette IT-Plattformen sowie Dienste für das "Internet der Dinge" offeriert. Die Rechenzentren stehen in Frankfurt am Main und bei Magdeburg. Neu ist ein Treuhänderkonzept, das verhindern soll, dass die US-Justiz unmittelbar auf Daten zugreifen kann.

\section{Messaging-App: Google bläst zur Aufholjagd}

Google macht WhatsApp und anderen Kurzmitteilungsdiensten Konkurrenz mit einer App mit eingebautem persönlichem Assistenten. Der zunächst in den USA gestartete Messenger "Allo" für Android und iOS enthält den neuen "Google Assistant". Nutzer können sich von ihm per Chat zum Beispiel Informationen zu Wetter, Nachrichten oder Reisemöglichkeiten anzeigen lassen oder ihm auch Wissensfragen stellen. Außerdem schlägt "Allo" in Unterhaltungen mit anderen Menschen mögliche passende Antworten vor. 